\title{
Prevalence and Correlates of Type D Personality among Survivors following Acute Myocardial Infarction in a Tertiary Care Center in South India
}

\author{
${ }^{1}$ Department of Psychiatry, Jawaharlal Institute of Post Graduate \\ Medical Education and Research, Puducherry, India \\ 2Department of Cardiology, Jawaharlal Institute of Post Graduate \\ Medical Education and Research, Puducherry, India
}

Anoop Gangadharan Pillai ${ }^{1}$ Vikas Menon ${ }^{1}$ Santhosh Satheesh ${ }^{2}$

\author{
Address for correspondence Vikas Menon, MD, Department of \\ Psychiatry, Jawaharlal Institute of Post Graduate Medical Education \\ and Research, Dhanvantri Nagar, Puducherry 605006, India \\ (e-mail: drvmenon@gmail.com).
}

Abstract
Keywords
- acute coronary
syndrome
- Asia
- coronary artery
disease
- myocardial infarction
- type D personality

Background Type D personality, described as a consistent tendency to experience increased levels of social inhibition and negative affectivity, has a robust association with negative outcomes following myocardial infarction (MI). However, little is known about its determinants in our setting. Our aim was to assess the prevalence and correlates of Type $\mathrm{D}$ personality among survivors following acute MI (AMI).

Methods Two hundred participants with AMI were recruited over an 18-month period. Type D scale-14 was used to evaluate the presence of Type D personality. Apart from sociodemographic factors, depression, anxiety, stress, coping, personality, quality of life, and perceived social support were assessed using standard measures.

Results Type D personality was present in $24 \%$ of samples $(n=48)$. In multivariate Poisson regression analysis, younger age (prevalence ratio [PR]: $0.950,95 \%$ confidence intervals [Cls]: 0.927-0.974), high depression (PR: 1.372, 95\% CI: 1.216-1.548), and low family support (PR: $0.898,95 \% \mathrm{Cl}$ : 0.849-0.949) emerged as independent predictors of Type D personality in participants with AMI.

Conclusion Type D personality is seen in a significant proportion of AMI, consistent with global literature. Our findings suggest that among Indian patients with AMI, the presence or absence of Type $\mathrm{D}$ personality may represent two distinct subpopulations.

\section{Introduction}

Globally, more than a quarter of all mortality can be attributed to coronary heart disease (CHD). ${ }^{1}$ India has witnessed a fourfold rise in the prevalence of coronary artery disease (CAD) in the past 40 years. $^{2}$ This has been ascribed to factors such as rapid urbanization and lifestyle changes due to epidemiologic transitions. With the disease having assumed epidemic proportions, clinicians and researchers are emphasizing primary and secondary prevention and treatment using both conventional and innovative strategies. ${ }^{3}$

The role of personality factors in mediating vulnerability to CHD first came into focus nearly 60 years ago. Primarily propelled by the seminal work of Friedman and Rosenman, ${ }^{4}$ much of the work in this area initially focused on Type A personality. ${ }^{5,6}$ Subsequent research, however, failed to show a robust association of Type A personality with outcomes in CHD. ${ }^{7,8}$ On the other hand, accumulating evidence pointed to a key role for broader psychosocial variables such as anxiety, depression, social isolation, and stress. ${ }^{9-13}$

Type D (distressed) personality was formulated by Denollet, ${ }^{14}$ in response to this growing body of evidence implicating negative affectivity (NA) such as depression and traits, such as social isolation, in the pathogenesis of CHD. Its taxonomy is based on a mixture of two relatively constant yet distinct factors-NA besides social inhibition (SI). Such persons have a consistent predisposition to undergo negative emotions (including depression, anxiety, anger, and hostility) across time and situations and also experience discomfort and insecurity in social situations. Further, they have a tendency to 
filter their emotional and social expression so as to avoid censure.

A fair amount of evidence links Type D personality to negative outcomes including mortality, emotional and behavioral sequelae (anxiety and depression), as well as impaired quality of life in CHD patients. ${ }^{15-17}$ However, there have been some conflicting reports too which suggested no association between Type D personality and absolute incident CHD risk among older adults without clinical features of $\mathrm{CHD}$ at intake. ${ }^{18}$ Given the strength of evidence linking Type D personality to poor outcomes in CHD, the availability of a short and convenient instrument to measure Type D scale (DS-14), and the high prevalence and more aggressive nature of CHD among ethnic South Asians, ${ }^{19}$ there seems to be a compelling case to study the prevalence and determinants of Type D among Indians with CHD about which there is little literature so far. This would give vital information about the extent of influence of personality, which may presumably vary across cultures, on CHD in the Indian setting. It would also, potentially, inform practical and innovative treatment strategies aimed at modifying long-term outcomes in CHD. Hence, our objective was to determine the frequency and correlates of Type D personality among a hospital-based sample of survivors following acute myocardial infarction (AMI).

\section{Methods}

\section{Setting and Design}

This was a cross-sectional study performed at a government medical college in Puducherry, South India, between August 2016 and December 2017. The centrally funded institution provides highly subsidized treatment to patients. Most service users are from Puducherry or nearby regions of Tamil Nadu. All patients with AMI are admitted in the cardiology and coronary care unit of the hospital. After stabilization, they are transferred to the ward from where they are eventually discharged.

\section{Subjects and Methods}

We included participants aged between 18 and 65 years who had a history of AMI (both ST elevation MI and non-ST elevation MI) diagnosed by a cardiologist. We excluded participants with psychosis and those who were unfamiliar with the local language (Tamil) or English. All consecutive patients presenting with AMI during the period of study were screened and those fulfilling the above criteria $(n=200)$ were selected for the research. All study participants gave written informed consent.

\section{Assessments}

We used a semistructured data sheet to collect pertinent sociodemographic and clinical details. This proforma also covered all the known CHD risk factors (such as diabetes, hypertension, smoking, and dyslipidemia). For diagnosis of metabolic syndrome (MetS), we used the International Diabetes Federation criteria, ${ }^{20}$ which requires a patient to have central obesity (defined as waist circumference of $\geq 80 \mathrm{~cm}$ for women and $\geq 90 \mathrm{~cm}$ for men among Asians) plus any two out of reduced high-density lipoprotein cholesterol, raised triglyceride, raised blood pressure, or raised fasting plasma glucose or any specific treatment for any of these abnormalities. Waist circumference was assessed using the National Institutes of Health protocol using an anthropometric tape. Body mass index was computed as weight (in kilograms) divided by height (in meter square).

Subsequently, all participants were also assessed on the following instruments:

1. DS-14 for Type D personality-This is the most widely used self-administered instrument for measuring Type D personality. ${ }^{14}$ All scale items (14) are scored on a 5-point Likert scale. The scale yields two subscale scores ranging from 0 to 28, namely SI and NA. Developers have suggested a cutoff of $\geq 10$ on both the subscales to diagnose Type D personality. We used a Tamil translated version of the DS-14 questionnaire following the guidelines suggested by the World Health Organization (WHO) for translating research tools ${ }^{21}$

2. Presumptive Stressful Life Events Scale-A 51-item self-administered scale which is specifically developed and validated for the Indian culture. ${ }^{22}$ The scale items are adapted from the larger Holmes and Rahe instrument. ${ }^{23}$ Both desirable and undesirable life events are included. For the present study, we used the sum of stressful events reported during the last 1 year

3. Coping Strategies Inventory Short Form-The scale has 16 items and is adapted from the larger 78-item scale. ${ }^{24}$ The scale seeks to tap individual coping responses to stressful situations. The reported strategies are first grouped into either emotion-focused or problem-focused coping; both these are further subclassified into engagement or disengagement form of coping. In this way, the instrument generates four subscale scores, each of which was analyzed separately.

4. Hospital Anxiety and Depression Scale (HADS)-A short, simple, easy to use screening instrument primarily devised to measure anxiety and depression in general medical population of patients. ${ }^{25}$ The scale has 14 items, 7 items each for anxiety and depression. We used the depression and anxiety subscale scores as continuous variables for the present study.

5. World Health Organization Quality of Life (WHOQOL) - BREF-This instrument is drawn from the larger World Health Organization Quality of Life - BREF (WHOQOL-BREF) ${ }^{26}$ and comprises 26 items that assess respondent satisfaction in four areas over the past 1 month: physical, psychological, social, and environmental health. Higher scores indicate better quality of life. Guidelines are available for converting scores in the individual domains to a more linear scale measuring 0 to 100 . This transformed score of each domain was used as a dependent variable. Domain-wise Cronbach's $\alpha$ values were $0.82,0.79,0.67$, and 0.78 for physical, psychological, social, and environmental domains, respectively, indicating acceptable internal consistency. 
6. Multidimensional Scale of Perceived Social Support-This is a widely used measure ${ }^{27}$ for subjectively assessed social support. The self-report measure has 12 items. Family, friends, and significant others are the three support sources assessed using the measure. Accordingly, the scale yields three subscale scores indicating the perceived magnitude of support from each of these sources. The scale has robust psychometric properties and acceptable internal consistency (Cronbach's $\alpha$ ranges from 0.81 to 0.98 ). We analyzed and used three subscale scores separately.

All the above instruments were applied only once at baseline, by the same rater. The research was approved by the Institute Ethics Committee.

\section{Statistical Analysis}

SPSS Inc. (Released 2008, SPSS Statistics for Windows, Version 17.0. Chicago, United States) was used for data analysis. Means with standard deviations or frequencies and percentages as appropriate were used to summarize continuous and discrete data, respectively. For comparison of variables between groups, Student's t-test or Mann-Whitney U-test and chi-square test were employed. To identify independent predictors of Type D personality, a multivariable analysis using generalized linear model (Poisson regression with robust variance estimator) was employed to model outcome data and yield conservative estimates of outcomes. We did not perform binary logistic regression as Type $D$ personality was not a rare outcome in our sample, and in such cases, this approach tends to overestimate odds ratios. A two-tailed $p$-value of $<0.05$ was considered significant. Statistical correction was not considered for multiple hypothesis testing as this was an exploratory work.

\section{Results}

\section{Sample Description and Frequency of Type $D$}

Age of included participants $(n=200)$ ranged between 21 and 65 years. The mean (standard deviation) age was 55.0 (12.1)years. Comorbid diabetes was present in 63 patients (31.5\%), while 59 (29.5\%) were suffering from concurrent hypertension. Alcohol use was reported by 82 (41\%) patients while 93 (46.5\%) were smokers. Type D personality was present in 48 (24\%) participants. Other demographic and clinical parameters are presented in - Table $\mathbf{1}$.

\section{Sociodemographic and Clinical Correlates of Type D Personality}

AMI patients with concurrent Type D personality were noted to be significantly younger, employed, and married, with a higher body mass index and a significantly greater family history of hypertension. None of the other variables differentiated the two groups (-Table 2 ).

\section{Scale Scores and Metabolic Syndrome Components between Groups}

Participants with AMI and Type D endorsed higher ratings on both anxiety and depression subscales of HADS. This group had also experienced greater number of stressful life events
Table 1 Baseline sociodemographic characteristics

\begin{tabular}{|c|c|}
\hline Variable & $\begin{array}{l}\text { Mean (SD) or } \\
\text { frequency (\%) }\end{array}$ \\
\hline Age & $55.0(12.1)$ \\
\hline \multicolumn{2}{|l|}{ Gender (\%) } \\
\hline Male & $142(71.0)$ \\
\hline Female & $58(29.0)$ \\
\hline \multicolumn{2}{|l|}{ Education (\%) } \\
\hline Less than or equal to 10 th grade & $168(84)$ \\
\hline More than 10th grade & $32(16)$ \\
\hline \multicolumn{2}{|l|}{ Occupation (\%) } \\
\hline Unemployed & $109(54.5)$ \\
\hline Employed & $91(45.5)$ \\
\hline \multicolumn{2}{|l|}{ Marital status (\%) } \\
\hline Single & $27(13.5)$ \\
\hline Married & $173(86.5)$ \\
\hline \multicolumn{2}{|l|}{ Past history of CAD (\%) } \\
\hline Yes & $11(5.5)$ \\
\hline No & $189(94.5)$ \\
\hline \multicolumn{2}{|l|}{ Family history of hypertension (\%) } \\
\hline Yes & $62(31)$ \\
\hline No & $138(69)$ \\
\hline \multicolumn{2}{|l|}{ Diagnosis (\%) } \\
\hline NSTEMI & $36(18)$ \\
\hline STEMI & $164(82)$ \\
\hline \multicolumn{2}{|l|}{ Metabolic syndrome (\%) } \\
\hline Yes & $75(37.5)$ \\
\hline No & $125(62.5)$ \\
\hline \multicolumn{2}{|l|}{ Type D personality (\%) } \\
\hline Yes & $48(24)$ \\
\hline No & $152(76)$ \\
\hline
\end{tabular}

Abbreviations: CAD, coronary artery disease; NSTEMI, non-ST elevation myocardial infarction; SD, standard deviation; STEMI, ST elevation myocardial infarction.

Note: All values are expressed as mean (SD) or frequency (\%).

in the last 1 year and reported higher trait neuroticism scores. Subscale scores on all four domains of coping and quality of life were also significantly lower for participants having Type D personality. The Type D group also reported lower levels on all three subscales of perceived social support. Among the MetS components, the only difference between the groups was the greater systolic blood pressure ratings among the Type D-positive group (-Table 3 ).

\section{Predictors of Type D Personality}

Poisson regression was performed using two sequential models with six and eight covariates, respectively. The first model was adjusted for six variables, namely age, occupation, depression scores, body mass index, problem-focused engagement 
Table 2 Sociodemographic and clinical variables between acute myocardial infarction patients with and without Type D personality

\begin{tabular}{|c|c|c|c|}
\hline Variable & $\begin{array}{l}\text { AMI with Type D }(n=48), \\
n(\%)\end{array}$ & $\begin{array}{l}\text { AMI without Type D }(n=152) \text {, } \\
n(\%)\end{array}$ & Comparison ( $p$-Value) \\
\hline Age & $43.4 \pm 5.9$ & $58.6 \pm 11.2$ & $t=9.014(<0.001)^{a}$ \\
\hline \multicolumn{4}{|l|}{ Gender } \\
\hline Male & $34(70.8)$ & $108(71.1)$ & \multirow{2}{*}{$\chi^{2}=0.977(1.000)$} \\
\hline Female & $14(29.2)$ & $44(28.9)$ & \\
\hline \multicolumn{4}{|l|}{ Education } \\
\hline$\leq 10$ th grade & $32(66.7)$ & $136(89.5)$ & \multirow{2}{*}{$\chi^{2}=0.015(0.902)$} \\
\hline$>10$ th grade & $16(33.3)$ & $16(10.5)$ & \\
\hline \multicolumn{4}{|l|}{ Occupation } \\
\hline Unemployed & $15(31.2)$ & $94(61.8)$ & \multirow{2}{*}{$\chi^{2}=13.768(<0.001)^{\mathrm{a}}$} \\
\hline Employed & $33(68.8)$ & $58(38.2)$ & \\
\hline \multicolumn{4}{|l|}{ Marital status } \\
\hline Single & $2(4.2)$ & $25(16.4)$ & \multirow{2}{*}{$\chi^{2}=4.711(0.030)^{\mathrm{a}}$} \\
\hline Married & $46(95.8)$ & $127(83.6)$ & \\
\hline \multicolumn{4}{|l|}{ Diagnosis } \\
\hline NSTEMI & $9(18.8)$ & $27(17.8)$ & \multirow{2}{*}{$\chi^{2}=0.024(0.887)$} \\
\hline STEMI & $39(81.2)$ & $125(82.2)$ & \\
\hline BMI & $26.7 \pm 2.4$ & $24.5 \pm 3.7$ & $t=-3.819(<0.001)^{\mathrm{a}}$ \\
\hline \multicolumn{4}{|c|}{ Metabolic syndrome } \\
\hline Yes & $30(62.5)$ & $95(62.5)$ & \multirow{2}{*}{$\chi^{2}=0.000(1.000)$} \\
\hline No & $18(37.5)$ & $57(37.5)$ & \\
\hline \multicolumn{4}{|l|}{ Alcohol use } \\
\hline Yes & $18(37.5)$ & $64(42.1)$ & \multirow{2}{*}{$\chi^{2}=0.320(0.572)$} \\
\hline No & $30(62.5)$ & $88(57.9)$ & \\
\hline \multicolumn{4}{|l|}{ Smoker } \\
\hline Yes & $25(52.1)$ & $68(44.7)$ & \multirow{2}{*}{$\chi^{2}=0.791(0.374)$} \\
\hline No & $23(47.9)$ & $84(55.3)$ & \\
\hline \multicolumn{4}{|c|}{ Family history of hypertension } \\
\hline Yes & $28(58.3)$ & $34(22.4)$ & \multirow{2}{*}{$\chi^{2}=22.060(<0.001)^{\mathrm{a}}$} \\
\hline No & $20(41.7)$ & $118(77.6)$ & \\
\hline
\end{tabular}

Abbreviations: AMI, acute myocardial infarction; BMI, body mass index; NSTEMI, non-ST elevation myocardial infarction; SD, standard deviation; STEMI, ST elevation myocardial infarction.

Note: All values are expressed as mean (SD) or frequency (\%).

asignificance at $p<0.05$.

subscale scores, and family support subscale scores. In the next model, apart from the aforementioned variables, we also additionally adjusted for family history of hypertension and neuroticism scores. In both the models, the results were similar (pseudo $R 2=0.41$ and 0.42 , respectively, for the six and eight variable models), and only three variables, namely age, depression, and family subscale of perceived social support, emerged as independent predictors of Type D. Output of the regression model with six covariates are shown in -Table 4 .

\section{Discussion}

We found that nearly a quarter (24\%) of participants with AMI were positive for Type D. Further, lower age, higher depression scores, and lower levels of perceived family support were independently associated with Type D personality among those with AMI. Prior research has shown considerable variations in proportions of Type D in CHD with figures ranging from 14 to $35.9 \%$. In one of the earliest such studies, de Jonge et $\mathrm{al}^{28}$ noted a prevalence rate of $18.7 \%$ for Type $\mathrm{D}$ among MI patients. More recently, Vukovic et $\mathrm{a}^{29}$ found that $34.2 \%$ of inpatients with CAD qualified for Type D personality.

Our numbers closely tally with other Asian studies from Korea $^{30}$ and China, ${ }^{31}$ which reported prevalence figures of 26.1 and 31.4\%, respectively, for Type D personality in CAD. These differences in prevalence rates may be attributed to two factors, namely the nature and disposition of the sample including age and gender distribution and cultural variations 
Table 3 Scale scores and metabolic syndrome components between acute myocardial infarction patients with and without Type D personality

\begin{tabular}{|c|c|c|c|}
\hline Variable & $\begin{array}{l}\text { AMI with Type D } \\
(n=48)\end{array}$ & $\begin{array}{l}\text { AMI without Type D } \\
(n=152)\end{array}$ & Comparison ( $p$-Value) \\
\hline HADS anxiety & $1.7 \pm 0.4$ & $0.2 \pm 0.4$ & $U=204.00(<0.001)^{\mathrm{a}}$ \\
\hline HADS depression & $0.9 \pm 0.5$ & $0.2 \pm 0.4$ & $U=713.00(<0.001)^{\mathrm{a}}$ \\
\hline Neuroticism score & $8.7 \pm 1.2$ & $2.6 \pm 1.8$ & $U=28.00(<0.001)^{\mathrm{a}}$ \\
\hline PSLES number of events & $7.0 \pm 2.2$ & $4.2 \pm 1.5$ & $U=1125.00(<0.001)^{\mathrm{a}}$ \\
\hline \multicolumn{4}{|l|}{ CSI-SF } \\
\hline Problem-focused engagement & $9.3 \pm 4.2$ & $15.3 \pm 2.7$ & $U=1092.00(<0.001)^{\mathrm{a}}$ \\
\hline Problem-focused disengagement & $9.1 \pm 2.7$ & $12.8 \pm 2.1$ & $U=1086.50(<0.001)^{\mathrm{a}}$ \\
\hline Emotion-focused engagement & $10.0 \pm 2.1$ & $12.9 \pm 1.8$ & $U=1022.00(<0.001)^{a}$ \\
\hline Emotion-focused disengagement & $7.8 \pm 1.8$ & $13.5 \pm 2.6$ & $U=476.00(<0.001)^{\mathrm{a}}$ \\
\hline \multicolumn{4}{|l|}{ WHOQOL-BREF } \\
\hline Physical domain & $41.7 \pm 4.4$ & $58.3 \pm 7.1$ & $U=191.00(<0.001)^{\mathrm{a}}$ \\
\hline Psychological domain & $47.6 \pm 9.3$ & $63.0 \pm 8.6$ & $U=753.00(<0.001)^{a}$ \\
\hline Social domain & $26.6 \pm 14.5$ & $63.9 \pm 12.5$ & $U=343.00(<0.001)^{\mathrm{a}}$ \\
\hline Environmental domain & $57.0 \pm 12.6$ & $69.9 \pm 6.9$ & $U=1294.00(<0.001)^{a}$ \\
\hline \multicolumn{4}{|l|}{ MDSPSS } \\
\hline Family & $14.5 \pm 4.5$ & $21.4 \pm 3.1$ & $U=1015.00(<0.001)^{\mathrm{a}}$ \\
\hline Friends & $12.5 \pm 2.2$ & $20.7 \pm 2.4$ & $U=24.50(<0.001)^{\mathrm{a}}$ \\
\hline Significant others & $13.5 \pm 1.9$ & $20.1 \pm 3.3$ & $U=486.50(<0.001)^{\mathrm{a}}$ \\
\hline Systolic BP & $121.4 \pm 13.4$ & $116.1 \pm 16.1$ & $U=2955.00(0.045)^{\mathrm{a}}$ \\
\hline Diastolic BP & $76.2 \pm 7.8$ & $75.8 \pm 9.3$ & $U=3471.50(0.611)$ \\
\hline Fasting sugar & $126.7 \pm 57.1$ & $112.5 \pm 37.2$ & $U=3150.50(0.154)$ \\
\hline \multicolumn{4}{|l|}{ Central obesity, n (\%) } \\
\hline Yes & $34(70.8)$ & $105(69.1)$ & \multirow[t]{2}{*}{$\chi^{2}=0.053(0.818)$} \\
\hline No & $14(29.2)$ & $47(30.9)$ & \\
\hline \multicolumn{4}{|l|}{ Hypertriglyceridemia, n (\%) } \\
\hline Yes & $41(85.4)$ & $125(82.2)$ & \multirow[t]{2}{*}{$\chi^{2}=0.261(0.609)$} \\
\hline No & $7(14.6)$ & $27(17.8)$ & \\
\hline \multicolumn{4}{|l|}{ HDL status, $n(\%)$} \\
\hline Low & $36(75.0)$ & $131(86.2)$ & \multirow[t]{2}{*}{$\chi^{2}=3.312(0.069)$} \\
\hline High & $12(25.0)$ & $21(13.8)$ & \\
\hline
\end{tabular}

Abbreviations: AMI, acute myocardial infarction; BP, blood pressure; CSI-SF, Coping Strategies Inventory Short Form; HADS, Hospital Anxiety and Depression Scale; HDL, high-density lipoprotein; MDSPSS, Multidimensional Scale of Perceived Social Support; PSLES, Presumptive Stressful Life Events Scale; WHOQoL-BREF, World Health Organization Quality of Life-BREF Scale.

Note: Hypertriglyceridemia when triglycerides $\geq 150 \mathrm{mg} / \mathrm{dL}$ or receiving treatment for the same, central obesity when waist circumference $\geq 90 \mathrm{~cm}$ for males and $\geq 80 \mathrm{~cm}$ for females, and HDL low when $\leq 40 \mathrm{mg} / \mathrm{dL}$ in males or $\leq 50 \mathrm{mg} / \mathrm{dL}$ in females or receiving treatment for the same.

asignificance at $p<0.05$.

in response patterns as has been pointed out earlier. ${ }^{32}$ Our sample mainly consisted of males, among whom overt expression of emotions and feelings is discouraged in the largely patriarchal Indian culture. ${ }^{33}$ Hence, their responses may have been more measured, which may have led to lower prevalence rates of type D noted in the present study. It also lends credence to the viewpoint of earlier researchers who have emphasized the need to evolve culture-specific cutoffs for DS.
We found that, apart from elevated systolic blood pressure and higher body mass index, none of the traditional cardiovascular risk factors differed between the two groups. These findings are consistent with the literature ${ }^{34,35}$ and suggest that the impact of Type D construct on cardiac health indices in participants with CAD is not mediated through alterations in medical risk factors. On the other hand, neuroinflammation and hypothalamic-pituitary-adrenal axis hyperactivity may mediate the link between Type D personality construct and CAD. ${ }^{36}$ 
Table 4 Poisson regression to identify predictors of Type D personality among acute myocardial infarction survivors

\begin{tabular}{|l|l|l|l|}
\hline Variable & Prevalence ratio & $95 \% \mathrm{Cl}$ & $p$-Value \\
\hline Age & 0.950 & $0.927-0.974$ & $<0.001^{\mathrm{b}}$ \\
\hline Occupation & 1.058 & $0.722-1.550$ & 0.774 \\
\hline HADS-depression & 1.372 & $1.216-1.548$ & $<0.001^{\mathrm{b}}$ \\
\hline BMI & 0.985 & $0.936-1.037$ & 0.579 \\
\hline PFE & 0.942 & $0.869-1.021$ & 0.144 \\
\hline MDSPSS-family & 0.898 & $0.849-0.949$ & $<0.001^{\mathrm{b}}$ \\
\hline
\end{tabular}

Abbreviations: AMI, acute myocardial infarction; BMI, body mass index; $\mathrm{Cl}$, confidence interval; HADS-depression, Depression subscale of the Hospital Anxiety and Depression Scale; MDSPSS, Multidimensional Scale of Perceived Social Support; PFE, problem-focused engagement.

aDepicts all covariates included in the model.

bignificant at $p<0.05$.

Type D personality in CAD correlated with elevated levels of psychological variables such as stress, anxiety, and depression. While this tallies with the available literature, ${ }^{37,38}$ evidence also suggests that Type $\mathrm{D}$ and depression may represent distinct but overlapping forms of distress. ${ }^{39}$ One can conclude that Type D may be a marker for potential psychological distress in CAD patients. CAD patients who also had concurrent Type D personality preferentially used emotion-focused coping strategies over problem-focused coping. However, coping styles did not remain significant in multivariable analysis. Only younger age, higher depression, and lower levels of perceived social support predicted Type $\mathrm{D}$ personality in our sample. Prior research, aimed at determining unique coping styles of CAD patients with Type $\mathrm{D}$, has been inconclusive. ${ }^{40}$

Taken together, it appears plausible that people with Type D personality may put themselves more at risk of experiencing stressful situations through their unique personality attributes. The effect of stress is compounded in these individuals by the use of maladaptive coping and poor social support. The resultant biological changes induced by the stressful experience, such as hypercortisolemia and inflammation, may contribute to the poorer clinical trajectories of Type D-positive CAD participants. Future longitudinal studies are required to support this hypothesis.

In our sample, there was no contrast between Type $\mathrm{D}$ and non-Type D groups on most components of MetS, except for elevated systolic blood pressure in Type D group. Few studies have examined this association in CAD participants, and to the best of our knowledge, no comparable data are available from Asian settings. Prior studies, on medical as well as nonmedical populations, ${ }^{41-43}$ noted a higher risk of MetS among participants with Type D. Significantly, none of these studies included CAD participants, and hence, the data are not directly comparable. Vukovic et al, who studied associations among Type D and MetS in participants with clinically stable CAD (including angina pectoris as well as $\mathrm{MI}$ ), found that hypercholesterolemia, hypertriglyceridemia, and hypertension were more common in Type D participants. ${ }^{29}$ Our study may have been underpowered to detect significant differences in MetS components between groups. Larger studies are clearly needed to elucidate the nature of association between personality factors and metabolic risk factors for CAD.
Our study findings need to be interpreted keeping its limitations in mind. First, this was a facility-based study performed among patients with AMI at a tertiary care center, and these results may not necessarily extend to other settings and presentations of CHD. Second, no causal inferences can be made among Type D and CAD owing to the crosssectional nature of the study. Third, the absence of a control group precludes comparisons with rates of Type D among the general public. Nevertheless, to our knowledge, this is the first systematic effort aimed at assessing Type D construct among Indian patients with MI. Our findings extend the relevance of Type D construct to the Indian culture and setting. Our findings also add to the limited literature on the connection between MetS components and Type D personality in CHD. Ethnically, South Asians have a higher vulnerability to CAD. ${ }^{19}$ Further, there is robust evidence linking Type $\mathrm{D}$ personality with adverse outcomes following CAD independent of several known risk factors such as hypertension and disease severity. ${ }^{44}$ Taken together with our findings, we recommend further investigation of the potential etiological contribution of Type D personality in causation of MI. We also recommend evaluating broad-based interventions, also targeting personality attributes in MI patients, such as cardiac rehabilitation programs, for which there is some

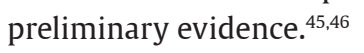

\section{Conclusion}

A significant percentage of participants attending a tertiary care hospital for AMI concurrently have Type D personality in our setting. Such individuals are more likely to be younger and more depressed and perceive less social support from traditional sources like the family. Detecting Type D in CAD may have important clinical and research implications, particularly among South Asians. Future research must examine the mechanisms linking Type D personality and CAD which may, potentially, yield new treatment targets to favorably modify the prognosis of these individuals.

\footnotetext{
Funding

None.

Conflict of Interest

None declared.
} 


\section{References}

1 World Health Organization, Preventing Chronic Diseases: A Vital Investment. Geneva: World Health Organization; 2005

2 Krishnan MN. Coronary heart disease and risk factors in India - on the brink of an epidemic? Indian Heart J 2012;64(4):364-367

3 Prabhakaran D, Jeemon P, Roy A. Cardiovascular diseases in India: current epidemiology and future directions. Circulation 2016;133(16):1605-1620

4 Friedman M, Rosenman RH. Overt behavior pattern in coronary disease. Detection of overt behavior pattern A in patients with coronary disease by a new psychophysiological procedure. JAMA 1960;173:1320-1325

5 Rosenman RH, Brand RJ, Jenkins D, Friedman M, Straus R, Wurm M. Coronary heart disease in Western Collaborative Group Study. Final follow-up experience of $81 / 2$ years. JAMA 1975;233(8):872-877

6 Kawachi I, Sparrow D, Kubzansky LD. Spiro A III, Vokonas PS, Weiss ST. Prospective study of a self-report type A scale and risk of coronary heart disease: test of the MMPI-2 type A scale. Circulation 1998;98(5):405-412

7 Rozanski A, Blumenthal JA, Kaplan J. Impact of psychological factors on the pathogenesis of cardiovascular disease and implications for therapy. Circulation 1999;99(16):2192-2217

8 Shekelle RB, Hulley SB, Neaton JD, et al. The MRFIT behavior pattern study. II. Type A behavior and incidence of coronary heart disease. Am J Epidemiol 1985;122(4):559-570

9 Ziegelstein RC, Bush DE, Fauerbach JA. Depression, adherence behavior, and coronary disease outcomes. Arch Intern Med 1998;158(7):808-809

10 Pignalberi C, Patti G, Chimenti C, Pasceri V, Maseri A. Role of different determinants of psychological distress in acute coronary syndromes. J Am Coll Cardiol 1998;32(3):613-619

11 Roest AM, Martens EJ, de Jonge P, Denollet J. Anxiety and risk of incident coronary heart disease: a meta-analysis. J Am Coll Cardiol 2010;56(1):38-46

12 Barth J, Schumacher M, Herrmann-Lingen C. Depression as a risk factor for mortality in patients with coronary heart disease: a meta-analysis. Psychosom Med 2004;66(6):802-813

13 Holt-Lunstad J, Smith TB. Loneliness and social isolation as risk factors for CVD: implications for evidence-based patient care and scientific inquiry. Heart 2016;102(13):987-989

14 Denollet J. DS14: standard assessment of negative affectivity, social inhibition, and Type D personality. Psychosom Med 2005;67(1):89-97

15 Al-Qezweny MN, Utens EM, Dulfer K, et al. The association between type $\mathrm{D}$ personality, and depression and anxiety ten years after PCI. Neth Heart J 2016;24(9):538-543

16 Denollet J, Pedersen SS, Vrints CJ, Conraads VM. Predictive value of social inhibition and negative affectivity for cardiovascular events and mortality in patients with coronary artery disease: the type D personality construct. Psychosom Med 2013;75(9):873-881

17 Saeed T, Niazi GS, Almas S. Type-D personality: a predictor of quality of life and coronary heart disease. East Mediterr Health J 2011;17(1):46-50

18 Larson NC, Barger SD, Sydeman SJ. Type D personality is not associated with coronary heart disease risk in a North American sample of retirement-aged adults. Int J Behav Med 2013;20(2):277-285

19 Narasimhan S, McKay K, Bainey KR. Coronary artery disease in South Asians. Cardiol Rev 2012;20(6):304-311

20 The IDF Consensus Worldwide Definition of the Metabolic Syndrome, Available at http://www.idf.org/webdata/docs/ IDF_Meta_def_final.pdf. Accessed May 01, 2018

21 World Health Organization | Process of Translation and Adaptation of Instruments. World Health Organization. Available at: http://www.who.int/substance_abuse/research_tools/translation/en. Accessed April 29, 2018

22 Singh G, Kaur D, Kaur H. Presumptive stressful life events scale (PSLES) - a new stressful life events scale for use in India. Indian J Psychiatry 1984;26(2):107-114

23 Holmes TH, Rahe RH. The social readjustment rating scale. J Psychosom Res 1967;11(2):213-218

24 Addison CC, Campbell-Jenkins BW, Sarpong DF, et al. Psychometric evaluation of a coping strategies inventory short-form (CSI-SF) in the Jackson heart study cohort. Int J Environ Res Public Health 2007;4(4):289-295

25 Zigmond AS, Snaith RP. The hospital anxiety and depression scale. Acta Psychiatr Scand 1983;67(6):361-370

26 Skevington SM, Lotfy M, O'Connell KA; WHOQOL Group. The World Health Organization's WHOQOL-BREF quality of life assessment: psychometric properties and results of the international field trial. A report from the WHOQOL group. Qual Life Res 2004;13(2):299-310

27 Zimet GD, Powell SS, Farley GK, Werkman S, Berkoff KA. Psychometric characteristics of the multidimensional scale of perceived social support. J Pers Assess 1990;55(3-4):610-617

28 de Jonge P, Denollet J, van Melle JP, et al. Associations of type-D personality and depression with somatic health in myocardial infarction patients. J Psychosom Res 2007;63(5):477-482

29 Vukovic O, Tosevski DL, Jasovic-Gasic M, et al. Type D personality in patients with coronary artery disease. Psychiatr Danub 2014;26(1):46-51

30 Lim HE, Lee MS, Ko YH, et al. Assessment of the type D personality construct in the Korean population: a validation study of the Korean DS14. J Korean Med Sci 2011;26(1):116-123

31 Yu XN, Zhang J, Liu X. Application of the Type D Scale (DS14) in Chinese coronary heart disease patients and healthy controls. J Psychosom Res 2008;65(6):595-601

32 Williams L, O'Connor RC, Howard S, et al. Type-D personality mechanisms of effect: the role of health-related behavior and social support. J Psychosom Res 2008;64(1):63-69

33 Aiyappa S, Acharya Y. Gender differences in emotional intelligence of adolescents. Int J Sci Res (Ahmedabad) 2014;3:525-526

34 Pedersen SS, Denollet J. Type D personality, cardiac events, and impaired quality of life: a review. Eur J Cardiovasc Prev Rehabil 2003;10(4):241-248

35 Svansdottir E, van den Broek KC, Karlsson HD, Gudnason T, Denollet J. Type D personality is associated with impaired psychological status and unhealthy lifestyle in Icelandic cardiac patients: a cross-sectional study. BMC Public Health 2012;12:42

36 Denollet J, Conraads VM. Type D personality and vulnerability to adverse outcomes in heart disease. Cleve Clin J Med 2011;(Suppl 1):S13-S19

37 Yu DS, Thompson DR, Yu CM, Pedersen SS, Denollet J. Validating the Type D personality construct in Chinese patients with coronary heart disease. J Psychosom Res 2010;69(2):111-118

38 Pelle AJ, Denollet J, Zwisler AD, Pedersen SS. Overlap and distinctiveness of psychological risk factors in patients with ischemic heart disease and chronic heart failure: are we there yet? J Affect Disord 2009;113(1-2):150-156

39 Denollet J, de Jonge P, Kuyper A, et al. Depression and Type D personality represent different forms of distress in the Myocardial INfarction and Depression - Intervention Trial (MIND-IT) Psychol Med 2009;39(5):749-756

40 Moryś JM, Bellwon J, Jeżewska M, Adamczyk K, Gruchała M. The evaluation of stress coping styles and type D personality in patients with coronary artery disease. Kardiol Pol 2015;73(7):557-566

41 Mommersteeg PM, Kupper N, Denollet J. Type D personality is associated with increased metabolic syndrome prevalence and 
an unhealthy lifestyle in a cross-sectional Dutch community sample. BMC Public Health 2010;10:714

42 Tziallas D, Kostapanos MS, Skapinakis P, et al. The association between Type D personality and the metabolic syndrome: a cross-sectional study in a University-based outpatient lipid clinic. BMC Res Notes 2011;4:105

43 Einvik G, Dammen T, Hrubos-Strøm H, et al. Prevalence of cardiovascular risk factors and concentration of C-reactive protein in Type D personality persons without cardiovascular disease. Eur J Cardiovasc Prev Rehabil 2011;18(3):504-509
44 Pedersen SS, Denollet J, Is type D personality here to stay? Emerging evidence across cardiovascular disease patient groups. Curr Cardiol Rev; 2006;2(3):205-213

45 Cao X, Wong EM, Chow Choi K, Cheng L, Ying Chair S. Interventions for cardiovascular patients with type D personality: a systematic review. Worldviews Evid Based Nurs 2016;13(4):314-323

46 Denollet J, Brutsaert DL. Reducing emotional distress improves prognosis in coronary heart disease: 9-year mortality in a clinical trial of rehabilitation. Circulation 2001;104(17):2018-2023 